\title{
Anthropic Principle Favors the Holographic Dark Energy
}

\author{
Qing-Guo Huang ${ }^{1}$ and Miao $\mathrm{Li}^{1,2}$ \\ 1 Interdisciplinary Center of Theoretical Studies \\ Academia Sinica, Beijing 100080, China \\ and \\ 2 Institute of Theoretical Physics \\ Academia Sinica, P. O. Box 2735 \\ Beijing 100080 \\ huangqg@itp.ac.cn \\ mli@itp.ac.cn
}

We discuss the anthropic principle when applied to the holographic dark energy. We find that if the amplitude of the density fluctution is variable, the holographic dark energy fares better than the cosmological constant. More generally, the anthropic predictions agree better with observation for dark energy with $w_{\Lambda}=p_{\Lambda} / \rho_{\Lambda}$ decreasing over time.

Oct. 2004 
The discovery of dark energy [1,2] can be regarded as one of the most significant discoveries in observational cosmology in the past several years. Although recent observations seem to indicate that the cosmological constant is a good candidate for dark energy, there is still plenty of room for imaginations, and the nature of dark energy remains one of the deepest mysteries crying for understanding and explanation. The problem of dark energy includes: Why the order of magnitude of the dark energy density is much smaller than quantum corrections in quantum field theory? And why it is the same order as today's critical energy density of our universe? The latter is called the cosmic coincidence problem.

Weinberg in 1987 proposed that the anthropic principle can be a good resolution to the cosmological constant problem [3], and some people even take his calculation as a prediction for the existence of a non-vanishing dark energy. The anthropic principle presumes that the problem of dark energy can never be posed if there are no astronomers in a subuniverse at all. Thus, according to this idea, the density of dark energy can take a range of values in different subuniverses and the probability distribution of its observed values is conditioned by the requirement that there must be someone to measure it. As a very interesting result, the anthropic argument predicts the existence of a small positive cosmological constant [3, [4], whose value is not far from the observed one.

In the inflation scenario, our observed universe is a tiny patch in the early universe and the whole space-time may contain many disconnected regions which will grow up to many subuniverses. This offers a framework for applying the anthropic principle. Recent developments in the string/M theory compactifications also favor the working of the anthropic principle, after all, there are too many vacua for us to select a unique or a few based on physical principles. It appears that the anthropic rule is the only hope to select a vacuum in the vast string theory landscape. On the other hand, there could be some dynamical processes connecting different vacua, for example see [5], again, the anthropic principle seems unavoidable.

However, the authors of [6] found that life may exist in universes with some of the cosmological parameters differing from those in our own subuniverse by several orders of magnitude, if larger density fluctuations than those in our universe are allowed. The quantitative analysis of this proposal is carried out in [7], and the result obtained suggests that the anthropic prediction for the cosmological constant is much weakened by allowing the variation of the amplitude of the primordial fluctuation power spectrum, a much larger cosmological constant is more favorable. The anthropic over-prediction of the cosmological constant is also investigated in [8]. If the cosmological constant is really a constant, and all 
the assumptions involved in the calculation of $[7,8]$ hold, this is really bad news for people believing that the anthropic principle is the resolution.

The purpose of the present note is to examine the same question in the framework of the holographic dark energy [9]. Since the holographic dark energy evolves in time, it is not surprising that our result differs significantly, and we find that the anthropic principle makes a better prediction here if dark energy is holographic. Put in another way, the anthropic argument favors the holographic dark energy, not the cosmological constant.

The non-extensive entropy bound $S \leq \pi M_{p}^{2} L^{2}$ on the total entropy $S$ in a volume $L^{3}$ proposed by Bekenstein et al. [10] suggests that quantum field theory breaks down in large scale. A. Cohen et al. [11] proposed a relationship between UV and IR cut-offs to rescue the local quantum field theory from the spelling of formation of black holes, this results in a up-bound on the zero-point energy density. We may view this as the holographic dark energy, the magnitude of this dark energy is consistent with the cosmological observations. However, Hsu recently pointed out that the equation of state is not correct for describing the accelerating expansion of our universe [12]. Very recently, one of us [9] suggested that we should use the proper size of the future event horizon of our universe to cut-off the large scale, resulting in a correct equation of state of dark energy. The density of the holographic dark energy in a spatially flat universe is introduced in [9] (For previous work, see [11]; for related work, see [13]15]; and for a work relating the holographic dark energy to low multipoles in CMB spectrum, see [16])

$$
\rho_{\Lambda}=3 d^{2} M_{p}^{2} R_{h}^{-2}
$$

where $M_{p}$ is the reduced Planck mass, $R_{h}$ is the proper size of the future event horizon $1 R_{h}=a(t) \int_{t}^{\infty} d t^{\prime} / a\left(t^{\prime}\right)$ and $d$ is a free parameter. In particular, the holographic dark energy behaves as a cosmological constant with $w_{\Lambda}=-1$ as $t \rightarrow \infty$ for $\mathrm{d}=1$. The equation of state of the holographic dark energy [9, 14] is

$$
w_{\Lambda}=-\frac{1}{3} \frac{d \ln \rho_{\Lambda}}{d \ln a}-1=-\frac{1}{3}\left(1+\frac{2}{d} \sqrt{\Omega_{\Lambda}}\right),
$$

and the evolution of $\Omega_{\Lambda}$ in a universe with matter and dark energy is governed by (as resulting from the Friedmann equation)

$$
\frac{d \Omega_{\Lambda}}{d \ln a}=\Omega_{\Lambda}\left(1-\Omega_{\Lambda}\right)\left(1+\frac{2}{d} \sqrt{\Omega_{\Lambda}}\right)
$$

1 Causality in the holographic dark energy model was discussed in [9] and cannot be understood completely. 
Solving this equation [15], we obtain

$$
\begin{aligned}
& \ln \Omega_{\Lambda}-\frac{d}{2+d} \ln \left(1-\sqrt{\Omega_{\Lambda}}\right)+\frac{d}{2-d} \ln \left(1+\sqrt{\Omega_{\Lambda}}\right)-\frac{8}{4-d^{2}} \ln \left(d+2 \sqrt{\Omega_{\Lambda}}\right) \\
& =-\ln (1+z)+y_{0} .
\end{aligned}
$$

Weinberg obtained an inequality between the density fluctuation and the cosmological constant in [3]. Given an over-density $\delta$, the cosmological constant can not be greater than a value depending on $\delta$ in order for this region to collapse to form a galaxy or a cluster of galaxies. In our case, the density of the holographic dark energy evolves during the evolution of universe, the result of [3] can not be used, we need to find the bound for the holographic dark energy independently. Following Peebles [17] and Weinberg [3], the density fluctuation in a universe with holographic dark energy can be modelled as a sphere within which there is a uniform excess density $\triangle \rho(t)$, and a gravitational field described by FRW metric with positive curvature constant $\triangle k>0$ and the scale factor $a(t)$. The evolution of the fluctuation is governed by the Friedmann equation

$$
\dot{a}^{2}+\triangle k=\frac{1}{3 M_{p}^{2}} a^{2}\left(\rho+\triangle \rho+\rho_{\Lambda}\right),
$$

where $\rho$ is the unperturbed cosmic mass density. The total matter density observes the mass conservation law thus

$$
a^{3}(\rho+\triangle \rho)=\text { const }
$$

In general as $t \rightarrow 0$, we have $\rho_{\Lambda} \ll \Delta \rho \ll \rho$. To the first order in the perturbation solution of the Friedmann equation, there is a relation between the perturbed curvature constant and density perturbation, the same as in [3]

$$
\triangle k=\frac{5}{9 M_{p}^{2}} a^{2}(\rho+\triangle \rho)^{2 / 3} \tilde{\rho}^{1 / 3},
$$

where

$$
\tilde{\rho}=\frac{(\triangle \rho)^{3}}{\rho^{2}},
$$

a quantity independent of time.

The R.H.S. of eq. (5) reaches its minimum when

$$
\frac{\rho+\triangle \rho}{\rho_{\Lambda}^{\min }}=\frac{1-\Omega_{\Lambda}^{\min }}{\Omega_{\Lambda}^{\min }}=-\left(3 w_{\Lambda}^{\min }+1\right) .
$$


Using eq. (2), we get

$$
\left(1+\frac{2}{d} \sqrt{\Omega_{\Lambda}^{\min }}\right) \Omega_{\Lambda}^{\min }=1
$$

where quantities with superscript min are the ones at the time when the R.H.S. of (5) reaches its minimum. In this paper we take a special case with $d=1$, then $\Omega_{\Lambda}^{\min } \simeq 0.432$ and $w_{\Lambda}^{\min } \simeq-0.772$.

Now, for this region to re-collapse, there must be a time when $\dot{a}=0$. For this to happen, the minimum of the R.H.S. of eq. (5) should be less than $\triangle k$. Thus we find a bound for the holographic dark energy

$$
\frac{1}{3 M_{p}^{2}} \frac{-3 w_{\Lambda}^{\min }}{\left(3 w_{\Lambda}^{\text {min }}+1\right)^{2 / 3}} a^{2}\left(\rho_{\Lambda}^{\min }\right)^{1 / 3}(\rho+\triangle \rho)^{2 / 3}<\triangle k
$$

Using eq. (7), the bound can be expressed as

$$
\rho_{\Lambda}^{\min }<\left(\frac{5}{3} \frac{\left(3 w_{\Lambda}^{\min }+1\right)^{2 / 3}}{-3 w_{\Lambda}^{\min }}\right)^{3} \tilde{\rho} \simeq 0.645 \tilde{\rho}
$$

For the cosmological constant case, $w_{\Lambda}=-1$ and $\rho_{\Lambda}<\frac{500}{729} \tilde{\rho}$, the same as [3].

We like to find a bound on the holographic energy density at the recombination time. The holographic dark energy density is not constant. Using eq. (2) and (3), the relation between the holographic dark energy density at the recombination time $\rho_{\Lambda}^{r e c}$ and $\rho_{\Lambda}^{\min }$ is found to be

$$
\begin{aligned}
& \ln \frac{\rho_{\Lambda}^{r e c}}{\rho_{\Lambda}^{\text {min }}}=-2\left(\frac{1-d}{2+d} \ln \frac{1-\sqrt{\Omega_{\Lambda}^{r e c}}}{1-\sqrt{\Omega_{\Lambda}^{m i n}}}+\frac{1+d}{2-d} \ln \frac{1+\sqrt{\Omega_{\Lambda}^{r e c}}}{1+\sqrt{\Omega_{\Lambda}^{m i n}}}\right. \\
& \left.-\frac{12}{4-d^{2}} \ln \frac{d+2 \sqrt{\Omega_{\Lambda}^{r e c}}}{d+2 \sqrt{\Omega_{\Lambda}^{m i n}}}+\ln \frac{\Omega_{\Lambda}^{r e c}}{\Omega_{\Lambda}^{\text {min }}}\right) .
\end{aligned}
$$

Since $\Omega_{\Lambda}^{r e c} \simeq \rho_{\Lambda}^{r e c} / \bar{\rho} \ll 1$, where $\bar{\rho}$ is the energy density of non-relativistic matter at recombination, for $d=1$, we have

$$
\rho_{\Lambda}^{m i n}=\left(1+\sqrt{\Omega_{\Lambda}^{m i n}}\right)^{-4}\left(1+2 \sqrt{\Omega_{\Lambda}^{m i n}}\right)^{8}\left(\Omega_{\Lambda}^{m i n}\right)^{-2}\left(\frac{\rho_{\Lambda}^{r e c}}{\bar{\rho}}\right)^{3} \bar{\rho} \simeq 585\left(\frac{\rho_{\Lambda}^{r e c}}{\bar{\rho}}\right)^{3} \bar{\rho} .
$$

Combining eq.(12) and (14), the bound of the energy density of the holographic dark energy at recombination is found to be

$$
\frac{\rho_{\Lambda}^{r e c}}{\bar{\rho}}<0.103 \delta
$$


where $\delta=\left.\frac{\triangle \rho}{\bar{\rho}}\right|_{r e c}$ is the value of the average fractional over-density at recombination. However, the bound for the cosmological constant is $\frac{\rho_{\Lambda}}{\bar{\rho}} \leq \frac{500}{729} \delta^{3}$. The anthropic bound for holographic dark energy is quite different from the cosmological constant case.

We assume that the geometry in a sub-universe in general is different from others and there should be a distribution of the holographic dark energy in the ensemble. According to the principle of Bayesian statistics, the probability distribution of the dark energy is

$$
P_{o b s}\left(\rho_{\Lambda}\right)=\frac{A\left(\rho_{\Lambda}\right) P\left(\rho_{\Lambda}\right)}{\int_{0}^{\infty} A\left(\rho_{\Lambda}\right) P\left(\rho_{\Lambda}\right) d \rho_{\Lambda}},
$$

where $A\left(\rho_{\Lambda}\right)$ is the mean number of astronomers who can measure the dark energy density in a subuniverse. Since $\rho_{\Lambda}$ is much smaller than energy scales in theory of elementary particle physics, we usually assume that the a priori probability distribution $P\left(\rho_{\Lambda}\right)$ is a constant (This point can be questioned if dark energy is not really a constant, since the underlying dynamics of the nonconstant dark energy may result in a nontrivial distribution for $\rho_{\Lambda}$ ). The authors of [4] propose that the number of astronomers $A\left(\rho_{\Lambda}\right)$ who can measure $\rho_{\Lambda}$ in a subuniverse should be proportional to the fraction $F\left(\rho_{\Lambda}\right)$ of baryonic matter incorporated in galaxies. We also adopt these assumptions, thus, the fraction of mass winding up in galaxies for the holographic dark model is

$$
F\left(x=\rho_{\Lambda}^{r e c} / \bar{\rho}\right)=\int_{\frac{x}{0.103}}^{\infty} d \delta \frac{\delta N(\delta)}{\frac{x}{0.103} \times \frac{1}{s}+\delta},
$$

where we assume the fluctuation distribution be Gaussian distribution

$$
N(\delta)=\frac{1}{\sigma}\left(\frac{2}{\pi}\right)^{1 / 2} \exp \left(-\frac{\delta^{2}}{2 \sigma^{2}}\right),
$$

and $s$ is the ratio of the volume of the over-density sphere to the volume of the underdensity shell surrounding the sphere. In this paper we set $s=1$. The relation (17) is the same as in the one in [4], except that the lower limit of the integral is set by the new inequality (15). This lower limit is quite different from the old one, since ours is a linear relation between $\delta$ and $x=\rho_{\Lambda} / \bar{\rho}$, while the old one is a linear relation between $\delta$ and $x^{3}$. This difference is the main source of our improved result over that of [7].

To compute the probabilities, we need to normalize (17). The normalization integral in eq. (17) can be calculated by interchanging the orders of integrations over $x$ and $\delta$ :

$$
\int_{0}^{\infty} F(x) d x=0.103 \times \ln 2\left(\frac{2}{\pi}\right)^{1 / 2} \sigma .
$$


Thus for a given energy density of the holographic dark energy $\rho_{\Lambda}^{r e c}=x \bar{\rho}$ at recombination, the normalized probability distribution becomes

$$
P_{o b s}(x)=\frac{1}{0.103 \times \ln 2\left(\frac{2}{\pi}\right)^{1 / 2} \sigma} \int_{\frac{x}{0.103}}^{\infty} d \delta \frac{\delta N(\delta)}{\frac{x}{0.103}+\delta} .
$$

And the probability of a holographic dark energy with energy density greater than $\rho_{\Lambda *}^{r e c}$ is

$$
P_{o b s}\left(x>x_{*}\right)=\frac{1}{\ln 2 \times \sigma^{2}} \int_{\frac{x_{*}}{0.103}}^{\infty} \delta e^{-\frac{\delta^{2}}{2 \sigma^{2}}} \ln \left(\frac{0.206 \delta}{x_{*}+0.103 \delta}\right) d \delta,
$$

where $x_{*}=\rho_{\Lambda *}^{r e c} / \bar{\rho}$. Using $\Omega_{\Lambda *}^{0}=0.75$ for $d=1$ in our subuniverse, we fix the parameter $y_{0}$ to be -1.67 in eq. (四). As a consequence, we find that at recombination with $z_{\text {rec }}^{*}=1089$ (the first year result of WMAP [2] $x_{*} \simeq \Omega_{\Lambda_{*}}^{r e c} \simeq 1.821 \times 10^{-4}$ in our subuniverse.

In order to calculate the probability distribution, we need to know the variance $\sigma$ as in $N(\delta)$. We follow [4],

$$
\sigma^{2}=\frac{1}{2 \pi^{2}} \int_{0}^{\infty} P(k) W^{2}(k R) k^{2} d k
$$

where $P(k)$ is the power spectrum at recombination, $W(x)=\exp \left(-x^{2} / 2\right)$ is a Gaussian window function and $R$ is the minimal size of structures that are relevant in providing environment for emergence of intelligence. The window function is used to filter the underlying density field to exclude the contribution from perturbations of small wavelengths. We do not really know the sufficient conditions for the formation of astronomers, thus we assume the order of the magnitude of $R$ be around $1 \mathrm{Mpc}$. After taking the evolution of the power spectrum into account, the authors of [4] find

$$
\sigma\left(z_{\text {rec }}\right)=\left(c_{100}\right)^{(n+3) / 2}\left(1+z_{r e c}\right)^{-1} \Gamma^{(n+3) / 2} \delta_{H} f\left(\Omega_{\Lambda}^{0}\right) K_{n}^{1 / 2}\left(q_{\max }\right),
$$

where $c_{100}=2997.9$ is the speed of light in units of $100 \mathrm{~km} / \mathrm{sec}, n$ is the index of the primordial power spectrum (here we assume a scale-invariant spectrum with $n=1$ ),

$$
\delta_{H}=1.94 \times 10^{-5}\left(\Omega_{m}^{0}\right)^{-0.785-0.05 \times \ln \Omega_{m}^{0}}
$$

is COBE normalized amplitude of fluctuations at horizon crossing, and

$$
\begin{aligned}
& f\left(\Omega_{\Lambda}^{0}\right)=\frac{6\left(\Omega_{\Lambda}^{0}\right)^{5 / 6}}{5\left(\Omega_{m}^{0}\right)^{1 / 3}}\left[\int_{0}^{\Omega_{\Lambda}^{0} / \Omega_{m}^{0}} \frac{d w}{w^{1 / 6}(1+w)^{3 / 2}}\right]^{-1}, \\
& \Gamma=\Omega_{m}^{0} h e^{-\Omega_{B}^{0}-\Omega_{B}^{0} / \Omega_{m}^{0}} \\
& q=\frac{k}{h \Gamma \mathrm{Mpc}^{-1}}, \\
& K_{n}\left(q_{\max }\right)=\int_{0}^{\infty} q^{n+2} T^{2}(q) W^{2}\left(2 \pi \frac{q}{q_{\max }}\right) d q \\
& T(q)=\frac{\ln (1+2.34 q)}{2.34 q}\left[1+3.89 q+(16.1 q)^{2}+(5.46 q)^{3}+(6.71 q)^{4}\right]^{-1 / 4},
\end{aligned}
$$


where $q_{\max }=2 \pi /(h \Gamma R \mathrm{Mpc})$.

We take $\Omega_{B *}^{0}=0.044, h_{*}=0.71, \Omega_{m *}^{0}=1-\Omega_{\Lambda *}^{0}=0.25$ in our subuniverse [2] [15]. As in [4], we first assume that the variance is the same in different subuniverses (this was latter challenged in [6] and [7]). Numerically $\sigma_{*}=2.397 \times 10^{-3}$ for $R=1 \mathrm{Mpc}$, with this input, we find $P_{o b s}\left(x>x_{*}\right)=28.2 \%$; for $R=2 \mathrm{Mpc}, \sigma_{*}=1.736 \times 10^{-3}, P_{o b s}\left(x>x_{*}\right)=16.4 \%$. Both results are satisfying according to the "principle of mediocrity".

Graesser et al. recently proposed, following [6], that there is a priori probability distribution for $\sigma$ [7]. They worked with an inflation model with potential of the inflaton $V(\phi)=\lambda \phi^{p}(p \geq 2)$, assuming the distribution of the coupling constant $\lambda$ be flat. The primordial power spectrum is proportional to the coupling constant $\lambda$. The power spectrum at recombination $P(k)$ in eq. (22) should be proportional to $\lambda$ also, therefore we have $\sigma^{2} \propto \lambda$. For a slow-roll inflation, $\lambda$ is a small parameter, we may assume a constant $a$ priori probability distribution of $\lambda$. 2 The probability distribution of $\sigma$ should be

$$
P(\sigma) \propto \frac{d \lambda}{d \sigma} \sim \sigma
$$

The probability distribution for $\rho_{\Lambda}=x \bar{\rho}$ becomes

$$
P_{o b s}(x) \sim \int d \sigma P(\sigma) F(x, \sigma) .
$$

After normalization, we have

$$
P_{o b s}\left(x>x_{*}\right)=\frac{1}{\ln 2 \times \int_{\sigma_{\min }}^{\sigma_{\max }} \sigma d \sigma} \int_{\sigma_{\min }}^{\sigma_{\max }} \frac{d \sigma}{\sigma} \int_{\frac{x_{*}}{0.103}}^{\infty} \delta e^{-\frac{\delta^{2}}{2 \sigma^{2}}} \ln \left(\frac{0.206 \delta}{x_{*}+0.103 \delta}\right) d \delta .
$$

In the holographic dark energy model, we find $P_{o b s}\left(x>x_{*}\right)=79.7 \%$ if $\sigma \in\left[0.1 \sigma_{*}, 10 \sigma_{*}\right]$, $P_{o b s}\left(x>x_{*}\right)=97.5 \%$ if $\sigma \in\left[0.01 \sigma_{*}, 100 \sigma_{*}\right]$, for $R=1 \mathrm{Mpc}$; and $P_{o b s}\left(x>x_{*}\right)=73.6 \%$ if $\sigma \in\left[0.1 \sigma_{*}, 10 \sigma_{*}\right], P_{o b s}\left(x>x_{*}\right)=96.6 \%$ if $\sigma \in\left[0.01 \sigma_{*}, 100 \sigma_{*}\right]$, for $R=2 \mathrm{Mpc}$. However for the cosmological constant, we have $P_{o b s}\left(x>x_{*}\right)=99.96 \%$ for $\sigma \in\left[0.1 \sigma_{*}, 10 \sigma_{*}\right]$ when $R=1 \mathrm{Mpc}$; and $P_{o b s}\left(x>x_{*}\right)=99.90 \%$ for $\sigma \in\left[0.1 \sigma_{*}, 10 \sigma_{*}\right]$ when $R=2 \mathrm{Mpc}$. The "principle of mediocrity" presumes that our universe is a typical one among those friendly to intelligence, thus probability $P_{o b s}\left(x>x_{*}\right)$ must be a number not too close to 1 or too close to 0. Comparing our result about the holographic dark energy to the one on the cosmological constant, it appears that the anthropic rule favors the holographic dark energy after we take a priori probability distribution for $\sigma$ into account.

2 The same argument could just as well be applied to $\lambda^{2}$ and the results will be different. 
The reason that the result on the holographic dark energy looks better than that on the cosmological constant is that the holographic dark energy evolves rather rapidly in the matter dominated era, so around the recombination time, dark energy is greater, and the lower bound on the mass perturbation to form structures is much larger-because of both the larger dark energy density and the modified lower bound relation.

The last point we want to discuss about is whether the a priori distribution of dark energy is flat or not. For a cosmological constant, we can imagine of no reason for a nontrivial distribution near zero. However, the holographic dark energy evolves, it may have a nontrivial distribution. According to eq. (2), $w_{\Lambda} \simeq-1 / 3$ if a component of energy other than the dark energy dominates the universe and $\rho_{\Lambda} \propto a^{-2}$. We assume that there was an inflation period and the inflation is driven by a inflaton with a potential $V(\phi)=\frac{1}{2} m^{2} \phi^{2}$. The COBE normalization results in $m=7.4 \times 10^{-6}$, here we always work with the unit in which the reduced Planck mass $M_{p}=1$. During the inflation era, the holographic dark energy may start with a value much greater than inflaton energy. The slow-roll inflation begins when the inflaton energy is comparable to the dark energy, and the latter is quickly red-shifted away. The holographic dark energy density at the end of inflation is roughly

$$
\rho_{\Lambda}^{e n d} \sim \frac{1}{2} m^{2} \phi_{i}^{2} e^{-2 N_{t o t}}
$$

where $\phi_{i}$ is the initial value of $\phi$ and $N_{t o t} \simeq \phi_{i}^{2} / 4-1 / 2$ is the total e-folding number. We see that the holographic energy really depends on the number of e-folds, and it will have a nontrivial distribution if $N_{t o t}$ is a random number. It is quite reasonable that we require the initial energy density of inflaton be less than $M_{p}^{4}$, which means $\phi_{i}^{2} \leq 2 / m^{2} \simeq$ $3.7 \times 10^{10}$. On the other hand, the condition for the end of inflation is $\phi^{2} \simeq 2$. Thus we have $2 \leq \phi_{i}^{2} \leq 3.7 \times 10^{10}$, and $\ln \rho_{\Lambda}^{\text {end }} \sim-\phi_{i}^{2} / 2$. Since $\rho_{\Lambda}^{r e c} \propto \rho_{\Lambda}^{\text {end }}$,

$$
d \phi_{i} \sim \frac{d \rho_{\Lambda}^{r e c}}{\rho_{\Lambda}^{r e c} \sqrt{\left|\ln \rho_{\Lambda}^{r e c}\right|}} .
$$

Assuming the a priori probability distribution of $\phi_{i}$ be a constant, we find probability distribution of dark energy

$$
P\left(\rho_{\Lambda}\right) \sim \frac{1}{\rho_{\Lambda}^{r e c} \sqrt{\left|\ln \rho_{\Lambda}^{r e c}\right|}} .
$$

This probability distribution favors small $\rho_{\Lambda}^{r e c}$, the integral over $\rho_{\Lambda}^{r e c}$ diverges near the origin, so there must be a cut-off for $\rho_{\Lambda}^{r e c}$. This cut-off corresponds to the upper bound on the initial value $\phi_{i}$ which in turn derives from the upper bound on the initial inflaton 
energy, thus, we have $\left(\rho_{\Lambda}^{\text {end }}\right)_{\text {min }} \simeq 10^{-8 \times 10^{9}}$, an extremely small value. Multiplying the weight function in eq. (31), we find that the anthropic argument favors an extremely small value of dark energy and this prior is ruled out be the observed larger $\rho_{\Lambda}$ at very high significance.

However, it is rather premature to discuss the nontrivial distribution of dark energy, since we do not know whether the flat measure $d \phi_{i}$ is correct, and since unknown physics in the inflation era and beyond can not be ignored.

To summarize, we find that if the amplitude of density fluctuation is variable, the anthropic consideration favors the holographic dark energy over the cosmological constant. It is possible that the holographic dark energy is not the only form of dark energy that fares better than the cosmological constant. A more general conclusion is that, the anthrophic predictions agree better with observation for a the state parameter $w_{\Lambda}$ decreasing over time.

Acknowledgments.

The research of Huang is supported by a grant from NSFC and a grant from China Postdoctoral Science Foundation. Li is supported by a grant from CAS and a grant from NSFC. 


\section{References}

[1] A. G. Riess et al., Astron. J. 116 (1998) 1009, astro-ph/9805201; S. Perlmutter et al., Astrophys. J. 517 (1999) 565, astro-ph/9812133; A. G. Riess et al., Astrophys. J. 607 (2004) 665, astro-ph/0403292; Y. Wang and M. Tegmark, Phys. Rev. Lett 92 (2004) 241302, astro-ph/0403292.

[2] C. L. Bennett et al., Astrophys. J. Suppl. 148 (2003) 1, astro-ph/0302207.

[3] S. Weinberg, Phys. Rev. Lett. 59 (1987) 2607.

[4] H. Martel, P. R. Shapiro, S. Weinberg, Astrophys. J. 492 (1998) 29, astro-ph/9701099.

[5] Y. S. Piao, hep-th/0407258.

[6] A. Aguirre, Phys. Rev. D. 64 (2001) 083508, astro-ph/0106143; M. J. Rees, astroph/0401424; M. Tegmark and M. J. Rees, Astrophs. J. 499 (1998) 526, astroph/9709058.

[7] M. L. Graesser, S. D. H. Hsu, A. Jenkins and M. B. Wise, hep-th/0407174.

[8] M. Tegmark, astro-ph/0410281.

[9] Miao Li, hep-th/0403127.

[10] J. D. Bekenstein, Phys. Rev. D 7, 2333 (1973); J. D. Bekenstein, Phys. Rev. D 9, 3292 (1974); J. D. Bekenstein, Phys. Rev. D 23, 287 (1981); J. D. Bekenstein, Phys. Rev. D 49, 1912 (1994); S. W. Hawking, Phys, Rev. D 13, 191 (1976); G. 't Hooft, gr-qc/9310026; L. Susskind, hep-th/9409089, J. Math. Phys 36, 6377 (1994).

[11] A. Cohen, D. Kaplan and A. Nelson, hep-th/9803132, Phys. Rev. Lett. 82 (1999) 4971;

S. Thomas, Phys. Rev. Lett. 89 (2002) 081301.

[12] S. D. H. Hsu, hep-th/0403052.

[13] Y. Gong, hep-th/0404030; M. Ito, hep-th/0405281; S. Hsu and A. Zee, hep-th/0406142;

E. V. Shuryak, hep-th/0407190; K. Ke and M. Li, hep-th/0407056; R. Horvat, astroph/0404204; B. Wang and E. Abdalla and R. K. Su, hep-th/0404057.

[14] Qing-Guo Huang, Miao Li, JCAP 0408 (2004) 013, astro-ph/0404229.

[15] Qing-Guo Huang, Yungui Gong, JCAP 0408 (2004) 006, astro-ph/0403590.

[16] K. Enqvist and M. S. Sloth, hep-th/0406019; K. Enqvist, S. Hannestad and M. S. Sloth, astro-ph/0409275; J. Shen, B. Wang, E. Abdalla and R. Su, hep-th/0412227; Y. Myung, hep-th/0412224; Y. Gong, B. Wang and Y. Zhang, hep-th/0412218.

[17] P. J. E. Peebles, Astrophys. J. 147 (1967) 859. 